\title{
A Modified Averaging Composite Implicit Iteration Process for Common Fixed Points of a Finite Family of $\boldsymbol{k}$-Strictly Asymptotically Pseudocontractive Mappings
}

\author{
Donatus Igbokwe, Oku Ini \\ Department of Mathematics, University of Uyo, Uyo, Nigeria \\ E-mail: \{igbokwedi,inioku\}@yahoo.com \\ Received April 4, 2011; revised June 3, 2011; accepted June 10, 2011
}

\begin{abstract}
The composite implicit iteration process introduced by Su and Li [J. Math. Anal. Appl. 320 (2006) 882-891] is modified. A strong convergence theorem for approximation of common fixed points of finite family of $k$-strictly asymptotically pseudo-contractive mappings is proved in Banach spaces using the modified iteration process.
\end{abstract}

Keywords: Implicit Iteration Process, $k$-Strictly Asymptotically Pseudo-Contractive Maps, Fixed Points

\section{Introduction and Preliminaries}

Let $E$ be an arbitrary real Banach space and let $J$ denote normalized duality mapping from $E$ into $2^{E^{*}}$ given by

$$
J(x)=\left\{f \in E^{*}:\langle x, f\rangle=\|x\|^{2} ;\|x\|^{2}=\|f\|^{2}\right\}
$$

where $E^{*}$ denotes the dual space of $E$ and $\langle$, denotes the generalized duality pairing. If $E^{*}$ is strictly convex, then $J$ is single-valued. In the sequel, we shall denote single-valued duality mappings by $j$. A mapping $T: K \rightarrow K$ is called $k$-strictly asymptotically pseudocontractive with sequence $\left\{a_{n}\right\} \subseteq[1, \infty)$,

$\lim _{n \rightarrow \infty} a_{n}=1$ (see, for example [1]) if for all $x, y \in K$, there exists $j(x-y) \in J(x-y)$ and a constant $k \in[0,1)$ such that

$$
\begin{aligned}
& \left\langle T^{n} x-T^{n} y, j(x-y)\right\rangle \\
& \leq \frac{1}{2}\left(1+a_{n}\right)\|x-y\|^{2}-\frac{1}{2}(1-k)\left\|x-T^{n} x-\left(y-T^{n} y\right)\right\|^{2}
\end{aligned}
$$

for all $n \in N$. If $I$ denotes the identity operator, then (1) can be written in the form

$$
\begin{aligned}
& \left\langle\left(I-T^{n}\right) x-\left(I-T^{n}\right) y, j(x-y)\right\rangle \\
& \geq \frac{1}{2}(1-k)\left\|\left(I-T^{n}\right) x-\left(I-T^{n}\right) y\right\|^{2}-\frac{1}{2}\left(a_{n}-1\right)\|x-y\|^{2}
\end{aligned}
$$

The class of $k$-strictly asymptotically pseudocontractive maps was first introduced in Hilbert spaces by Qihou [2]. In Hilbert spaces, $j$ is the identity and it is shown in Osilike [3] that (1) (and hence (2)) is equivalent to the the inequality

$$
\left\|T^{n}-T^{n} y\right\|^{2} \leq a_{n}\|x-y\|^{2}+k\left\|\left(I-T^{n}\right) x-\left(I-T^{n}\right) y\right\|^{2}
$$

which is the inequality considered by Qihou [2].

A mapping $T$ with domain $D(T)$ and range $R(T)$ in $E$ is called strictly pseudo-contractive in the terminology of Browder and Petryshyn [4] if there exist $\lambda>0$ such that

$$
\langle T x-T y, j(x-y)\rangle \leq\|x-y\|^{2}-\lambda\|x-y-(T x-T y)\|^{2}
$$

for all $x, y \in D(T)$ and for all $j(x-y) \in J(x-y)$. Without loss of generality we may assume $\lambda \in(0,1)$. If I denotes the identity operator, then (1) can be written in the form

$$
\langle(I-T) x-(I-T) y, j(x-y)\rangle \geq\|(I-T) x-(I-T) y\|^{2}
$$

In the Hilbert space $H$, (4) (and hence (5)) is equivalent to the inequality

$$
\begin{aligned}
& \|T x-T y\|^{2} \leq\|x-y\|^{2}+k\|(I-T) x-(I-T) y\|^{2} \\
& k=(1-\lambda)<1
\end{aligned}
$$

and we can assume also that $k \geq 0$, so that $k \in[0,1]$. 
It is shown in [5] that a strictly pseudocontractive map is $L$-Lipschitzian $(\|T x-T y\| \leq\|x-y\|$, for all $x, y \in D(T)$ and for some $L>0$ ). It is also shown in [3] that a $k$-strictly asymptotically pseudocontractive mapping is uniformly $L$-Lipschitzian (i.e. for some $L>0$, $\left\|T^{n} x-T^{n} y\right\| \leq L\|x-y\|$, for all $x, y \in K$ and $\left.n \in N\right)$. The class of $k$-strictly asymptotically pseudocontractive mappings and the class of strictly pseudo-contractive mappings are independent (see [1]). The class of $k$-strictly asymptotically pseudocontractive mappings is a natural extension of the class of asymptotically nonexpansive mappings (i.e. mappings $T: K \rightarrow K$ such that

$$
\left\|T^{n} x-T^{n} y\right\| \leq a_{n}\|x-y\| \forall n \geq 1, \forall x, y \in K
$$

and for some sequence $\left\{a_{n}\right\} \subseteq[1, \infty)$ such that $\lim _{n \rightarrow \infty} a_{n}=1$.) If $k=0$, we have from (3) (and hence (1) ) that $T$ is asymptotically nonexpansive. In fact, an asymptotically nonexpansive map is 0 - strictly asymptotically pseudocontractive (see Remark 1 [6]). $T$ is called asymptotically quasi-nonexpansive if there exists a sequence $\left\{a_{n}\right\} \subseteq[1, \infty)$ such that $\lim _{n \rightarrow \infty} a_{n}=1$, and

$$
\left\|T^{n} x-p\right\| \leq a_{n}\|x-p\|, \forall n \geq 1
$$

for all $x \in K$ and $p \in F(T)=\{x \in K: T x=x\}$

In [7], $\mathrm{Xu}$ and Ori introduced an implicit iteration process and proved weak convergence theorem for approximation of common fixed points of a finite family of nonexpansive mappings (i.e. a subclass of asympto-

$$
\begin{array}{ccc}
x_{1} & = & \alpha_{1} x_{0}+\left(1-\alpha_{1}\right) T_{1} y_{1}, \\
x_{2} & = & \alpha_{2} x_{1}+\left(1-\alpha_{2}\right) T_{2} y_{2}, \\
\vdots & & \\
x_{N} & = & \alpha_{N} x_{N-1}+\left(1-\alpha_{N}\right) T_{N} y_{N}, \\
x_{N+1} & = & \alpha_{N+1} x_{N}+\left(1-\alpha_{N+1}\right) T_{1}^{2} y_{N+1}, \\
x_{N+2} & = & \alpha_{\alpha_{n+2}} x_{N+1}+\left(1-\alpha_{N+2}\right) T_{2}^{2} y_{N+2}, \\
\vdots & & \\
x_{2 N} & = & \alpha_{2 N} x_{2 N-1}+\left(1-\alpha_{2 N}\right) T_{N}^{2} y_{2 N}, \\
x_{2 N+1} & = & \alpha_{2 N+1} x_{2 N}+\left(1-\alpha_{2 N+1}\right) T_{1}^{3} y_{2 N+1}, \\
x_{2 N+2} & = & \alpha_{2 N+2} x_{2 N+1}+\left(1-\alpha_{2 N+2}\right) T_{2}^{3} y_{2 N+2}, \\
\vdots & &
\end{array}
$$

Our iteration process can be expressed in a compact form as

$$
\left.\begin{array}{l}
x_{n}=\alpha_{n} x_{n-1}+\left(1-\alpha_{n}\right) T_{i}^{k} y_{n} \\
y_{n}=\beta_{n} x_{n-1}+\left(1-\beta_{n}\right) T_{i}^{k} x_{n}
\end{array}\right\} n \geq 1
$$

where $n=(k-1) N+i, i=\{1,2, \cdots, N\}$. Observe that if $T: K \rightarrow K$ is $k$-strictly asymptotically pseudocon- tically nonexpansive mappings for which

$\|T x-T y\| \leq\|x-y\|, \quad \forall x, y \in K)$. In [8], Osilike extended the results of [7] from nonexpansive mappings to strictly pseudocontractive mappings. In [9], Su and Li introduced a new implicit iteration process and called it composite implicit iteration process. Using the new implicit iteration process, they proved the results established by Osilike in [8]. In compact form, the composite iteration process introduced in [9] is the sequence $\left\{x_{n}\right\}$ generated from arbitrary $x_{0} \in K$ by

$$
\left.\begin{array}{l}
x_{n}=\alpha_{n} x_{n-1}+\left(1-\alpha_{n}\right) T_{n} y_{n} \\
y_{n}=\beta_{n} x_{n-1}+\left(1-\beta_{n}\right) T_{n} x_{n}
\end{array}\right\}
$$

where $\left\{\alpha_{n}\right\},\left\{\beta_{n}\right\} \subseteq[0,1]$. In [10] Sun modified the implicit iteration process of Xu and Ori and applied the modified iteration process for the approximation of fixed points of a finite family of asymptotically quasi-nonexpansive maps. In compact form, the modified implicit iteration process of Sun is the sequence $\left\{x_{n}\right\}$ generated from arbitrary $x_{0} \in K$ by

$$
x_{n}=\alpha_{n} x_{n-1}+\left(1-\alpha_{n}\right) T_{i}^{k} x_{n}, n \geq 1
$$

where $n=(k-1) N+i, i \in I=\{1,2, \cdots, N\}$.

In this paper, we modify (9) as follows. Let $K$ be a nonempty closed convex subset of $E,\left\{T_{i}\right\}_{i=1}^{N}$ a finite family of $k$-strictly asymptotically pseudocontractive self-maps of $K$, then for $x_{0} \in K$ and $\left\{\alpha_{n}\right\},\left\{\beta_{n}\right\} \subset[0,1]$.

$$
\begin{array}{ccc}
y_{1} & = & \beta_{1} x_{0}+\left(1-\beta_{1}\right) T_{1} x_{1} \\
y_{2} & = & \beta_{2} x_{1}+\left(1-\beta_{2}\right) T_{2} x_{2} \\
\vdots & & \\
y_{N} & = & \beta_{N} x_{N-1}+\left(1-\beta_{N}\right) T_{N} x_{N} \\
y_{N+1} & = & \beta_{N+1} x_{N}+\left(1-\beta_{N+1}\right) T_{1}^{2} x_{N+1} \\
y_{N+2} & = & \beta_{N+2} X_{N}+\left(1-\beta_{N+2}\right) T_{N}^{2} x_{N+2} \\
\vdots & = & \\
y_{2 N} & = & \beta_{2 N} x_{2 N-1}+\left(1-\beta_{2 N}\right) T_{N}^{2} x_{2 N} \\
y_{2 N+1} & = & \beta_{2 N+1} x_{2 N}+\left(1-\beta_{2 N+1}\right) T_{1}^{3} x_{2 N+1} \\
y_{2 N+1} & = & \beta_{2 N+2} x_{2 N+1}+\left(1-\beta_{2 N+2}\right) T_{2}^{3} x_{2 N+2} \\
\vdots & &
\end{array}
$$

tractive mapping with sequence $\left\{a_{n}\right\} \subseteq[1, \infty)$ such that $\lim _{n \rightarrow \infty} a_{n}=1$, then for every fixed $u \in K$ and $t, s \in\{L /(1+L), 1\}$, the operator $S_{t, s, n}: K \rightarrow K$ defined for all $x \in K$ by $S_{t, s, n} x=t u(1-t) T^{n}\left(s u+(1-s) T^{n} x\right)$ satisfies $\left\|S_{t, s, n} x-S_{t, s, n} y\right\| \leq(1-t)(1-s) L^{2}\|x-y\|$, $\forall x, y \in K$. Since $(1-t)(1-s) L^{2} \in(0,1)$, it follows that 
$S_{t, s, n}$ is a contraction map and hence has a unique fixed point $x_{t, s, n}$ in $K$. This implies that there exists a unique $x_{t, s, n} \in K$ such that $x_{t, s, n}=t u+(1-t) T^{n}\left(s u+(1-s) T^{n} x_{t, s, n}\right)$. Thus our modified composite implicit iteration process (11) is defined in $K$ for the family $\left\{T_{i}\right\}_{i=1}^{N}$ of $N \quad k$-strictly asymptotically pseudocontractive self maps of a nonempty convex subset $K$ of a Banach space provided $\alpha_{n}, \beta_{n} \in(\eta, 1)$ where $\eta=L /(1+L)$ and

\section{$L=\max _{1 \leq N}\left\{L_{i}\right\}$.}

The purpose of this paper is to study the convergence of the new modified averaging implicit iteration scheme (11) to a common fixed point of a finite family of $k$ - strictly asymptotically pseudocontractive maps in arbitrary Banach spaces. The results presented in this paper, generalize the result of $\mathrm{Su}$ and $\mathrm{Li}$ [9] and several others in the literature (see for example [8], [11], [10], [7]).

In the sequel, we shall need the following:

Lemma 1.1 OAA ([3], p. 80):

Let $\left\{a_{n}\right\},\left\{b_{n}\right\}$ and $\left\{\delta_{n}\right\}$ be three sequences of nonnegative real numbers satisfying the inequality

$$
a_{n+1} \leq\left(1+b_{n}\right) a_{n}+\delta_{n}, n \geq 1
$$

If $\sum \delta_{n}<\infty$ and $\sum b_{n}<\infty$ then $\lim _{n \rightarrow \infty} a_{n}$ exists. If in addition $\left\{a_{n}\right\}$ has a subsequence which converges strongly to zero, then $\lim _{n \rightarrow \infty} a_{n}=0$.

Definition 1.1 [12] A bounded convex subset $K$ of a real Banach space $E$ is said to have normal structure if every nontrivial convex subset $C$ of $K$ contains at least one nondimetrial point. That is, there exists $x_{0} \in E$ such that

$$
\sup \left\{\left\|x_{0}-x\right\|: x \in C\right\}<\sup \{\|x-y\|: x, y \in C=d(C)\}
$$

where $d(C)$ is the diameter of $C$

Every uniformly convex Banach space and every compact convex subset $K$ of a Banach space $E$ has normal structure. For the definition of modulus of convexity of $E$ and the characteristic of convexity $\varepsilon_{0}$ of $E$, see [13].

Theorem 1.1 ([13] Corollary 3.6)

Let $E$ be a real Banch space with normal structure $N(E)>\max \left(1, \varepsilon_{0}\right), \quad \varepsilon_{0}>0, K$ a nonempty bounded closed convex subset of $E$ and $T: K \rightarrow K$ a uniformly $L$-Lipschitzian mapping with $L<\alpha, \alpha>1$. Then $T$ has a fixed point.

\section{Main Results}

Lemma 2.1 Let $E$ be a real Banach space with normal structure $N(E)>\max \left(1, \varepsilon_{0}\right)$ and let $K$ be a nonempty closed convex subset of $E$. Let $\left\{T_{i}\right\}_{i=1}^{N}$ be $N k_{i}-$ strictly asymptotically pseudo-contractive self-maps of $K$ with sequences $\left\{a_{i n}\right\} \subseteq[1, \infty)$ such that

$\sum_{n=1}^{\infty}\left(a_{i n}-1\right)<\infty$, and let $F=\cap F\left(T_{i}\right) \neq \varnothing$. Let $\left\{\alpha_{n}\right\}$, $\left\{\beta_{n}\right\} \subset(\eta, 1)$ be two real sequences satisfying the conditions:

(i) $\sum_{n=1}^{\infty}\left(1-a_{n}\right)=\infty$, (ii) $\sum_{n=1}^{\infty}\left(1-\alpha_{n}\right)^{2}<\infty$,

(iii) $\sum_{n=1}^{\infty}\left(1-\beta_{n}\right)<\infty$, (iv) $\left(1-\alpha_{n}\right)\left(1-\beta_{n}\right) L^{2}<1$, where $\eta=L /(1+L)$ and $L=\max _{1 \leq i \leq N}\left\{L_{i}\right\}, L_{i}$ the Lipschitzian constants of $\left\{T_{i}\right\}_{i=1}^{N}$. Let $\left\{x_{n}\right\}$ be the implicit iteration sequence generated by (11). Then

(i) $\lim _{n \rightarrow \infty}\left\|x_{n}-p\right\|$ exists for all $p \in F$.

(ii) $d\left(x_{n}, F\right)$ exists, where

$d\left(x_{n}, F\right)=\inf _{p \in F}\left\|x_{n}-p\right\|$

(iii) $\liminf _{n \rightarrow \infty}\left\|x_{n}-T_{n} x_{n}\right\|=0$.

Proof

The existence of fixed point follows from Theorem 1.1. We shall use the well known inequality (see for example $[7,14])$

$$
\|x+y\|^{2} \leq\|x\|^{2}+\langle y, j(x-y)\rangle
$$

which holds for all $x, y \in E$ and for all $j(x-y) \in J(x-y)$. Let $p \in F$, then using (11) and (13) we obtain

$$
\begin{aligned}
& \left\|x_{n}-p\right\|^{2}=\left\|\alpha_{n}\left(x_{n-1}-p\right)+\left(1-\alpha_{n}\right)\left(T_{i}^{k} y_{n}-p\right)\right\|^{2} \\
& \leq \alpha_{n}^{2}\left\|x_{n-1}-p\right\|^{2}+2\left(1-\alpha_{n}\right)\left\langle T_{i}^{k} y_{n}-p, j\left(x_{n}-p\right)\right\rangle \\
& =\alpha_{n}^{2}\left\|x_{n-1}-p\right\|^{2}+2\left(1-\alpha_{n}\right) \\
& \cdot\left[\left\langle T_{i}^{k} y_{n}-T_{i}^{k} x_{n}, j\left(x_{n}-p\right)\right\rangle+\left\langle T_{i}^{k} x_{n}, j\left(x_{n}-p\right)\right\rangle\right] \\
& \leq \alpha_{n}^{2}\left\|x_{n-1}-p\right\|^{2}+2\left(1-\alpha_{n}\right) \\
& \cdot\left[L\left\|y_{n}-x_{n}\right\|\left\|x_{n}-p\right\|+\left\|x_{n}-p\right\|^{2}\right] \\
& -2\left(1-\alpha_{n}\right)\left\langle x_{n}-T_{i}^{k} x_{n}, j\left(x_{n}-p\right)\right\rangle
\end{aligned}
$$

Since each $T_{i}: K \rightarrow K, \quad i \in I$, is $k_{i}-$ strictly asymptotically pseudocontractive, then

$$
\begin{aligned}
& \left\langle\left(I-T_{i}^{n}\right) x-\left(I-T_{i}^{n}\right) y, j(x-y)\right\rangle \\
& \geq \frac{1}{2}\left(1-k_{i}\right)\left\|x-T_{i}^{n} x-\left(y-T_{i}^{n} y\right)\right\|^{2}-\frac{1}{2}\left(a_{i m}-1\right)\|x-y\|^{2}
\end{aligned}
$$


$k_{i} \in[0,1)$. Let $k=\min _{1 \leq i \leq N}\left\{k_{i}\right\}$. Then

$$
\begin{aligned}
& \left\langle\left(I-T_{i}^{n}\right) x-\left(I-T_{i}^{n}\right) y, j(x-y)\right\rangle \\
& \geq \frac{1}{2}(1-k)\left\|x-T_{i}^{n} x-\left(y-T_{i}^{n} y\right)\right\|^{2}-\frac{1}{2}\left(a_{i m}-1\right)\|x-y\|^{2}
\end{aligned}
$$

Thus it follows from (14) that

$$
\begin{aligned}
\left\|x_{n}-p\right\|^{2} & \leq \alpha_{n}^{2}\left\|x_{n-1}-p\right\|^{2}+\left(1-\alpha_{n}\right) L\left\|y_{n}-x_{n}\right\|\left\|x_{n}-p\right\| \\
& +\left(1-\alpha_{n}\right)\left[2+\left(\alpha_{n}-1\right)\right]\left\|x_{n}-p\right\|^{2} \\
& -(1-k)\left(1-\alpha_{n}\right)\left\|x_{n}-T_{i}^{k} x_{n}\right\|^{2}
\end{aligned}
$$

Observe that

$\left\|y_{n}-x_{n}\right\| \leq \beta_{n}\left(1-\alpha_{n}\right)\left\|T_{i}^{k} y_{n}-x_{n-1}\right\|+\left(1-\beta_{n}\right)\left\|x_{n}-T_{i}^{k} x_{n}\right\|$

$$
\left\|T_{i}^{k} y_{n}-x_{n-1}\right\| \leq\left(L \beta_{n}+1\right)\left\|x_{n-1}-p\right\|+L^{2}\left(1-\beta_{n}\right)\left\|x_{n}-p\right\|
$$

and

$$
\left\|x_{n}-T_{i}^{k} x_{n}\right\| \leq(L+1)\left\|x_{n}-p\right\|
$$

Substituting (16)-(18) into (15), we obtain

$$
\begin{aligned}
& {\left[1-2\left(1-\alpha_{n}\right)^{2} L^{3} \beta_{n}\left(1-\beta_{n}\right)-2\left(1-\alpha_{n}\right)\left(1-\beta_{n}\right) L(L+1)\right.} \\
& \left.-\left(1-\alpha_{n}\right)\left(2+\left(a_{i k}-1\right)\right)\right]\left\|x_{n}-p\right\|^{2} \\
& \leq \alpha_{n}^{2}\left\|x_{n-1}-p\right\|^{2}+2\left(1-\alpha_{n}\right)^{2} L \beta_{n}\left(L \beta_{n}+1\right) \\
& \cdot\left\|x_{n-1}-p\right\|\left\|x_{n}-p\right\|-(1-k)\left(1-\alpha_{n}\right)\left\|x_{n}-T_{i}^{k} x_{n}\right\|^{2}
\end{aligned}
$$

Observe that $\left(a_{i k}-1\right) \leq\left(a_{i n}-1\right), \forall k=n$, since $n=(k-1) N+i, \forall i \in I=\{1,2, \cdots, N\}$. Setting

$$
\begin{aligned}
b_{n} & =2\left(1-\alpha_{n}\right)^{2} L^{2} \beta_{n}\left(1-\beta_{n}\right) \\
& +2\left(1-\alpha_{n}\right)^{2}\left(1-\beta_{n}\right) L(L+1)+\left(1-\alpha_{n}\right)\left(a_{n}-1\right)
\end{aligned}
$$

then it follows from (19) that

$$
\begin{aligned}
\left\|x_{n}-p\right\|^{2} & \leq\left[1+\frac{\left(1-\alpha_{n}\right)^{2}+b_{n}}{1-2\left(1-\alpha_{n}\right)-b_{n}}\right]\left\|x_{n-1}-p\right\|^{2} \\
& +\left[\frac{2\left(1-\alpha_{n}\right)^{2} L \beta_{n}\left(L \beta_{n}+1\right)}{1-2\left(1-\alpha_{n}\right)-b_{n}}\right]\left\|x_{n-1}-p\right\|\left\|x_{n}-p\right\| \\
& -\left[\frac{\left(1-\alpha_{n}\right)(1-k)}{1-2\left(1-\alpha_{n}\right)-b_{n}}\right]\left\|x_{n}-T_{i}^{k} x_{n}\right\|^{2}
\end{aligned}
$$

Since

$$
\begin{aligned}
& 1-2\left(1-\alpha_{n}\right)-b_{n} \\
& =1-\left(1-\alpha_{n}\right)\left[2+\left(a_{i n}-1\right)\right. \\
& \left.+2\left(1-\alpha_{n}\right) L^{3} \beta_{n}\left(1-\beta_{n}\right)+2\left(1-\beta_{n}\right) L(L+1)\right]
\end{aligned}
$$

and $\left\{\alpha_{n}\right\}\left\{\beta_{n}\right\} \subseteq(\eta, 1)$, then we obtain that

$$
\begin{aligned}
& 2+\left(a_{i n}-1\right)+2\left(1-\alpha_{n}\right) L^{3} \beta_{n}\left(1-\beta_{n}\right)+2\left(1-\beta_{n}\right) L(L+1) \\
& \leq 2+\left(a_{m}-1\right)+2 L^{3}+2 L(L+1)
\end{aligned}
$$

Setting $M_{1}=2+2 L^{3}+2 L(L+1)$, then there must exist a natural number $N_{1}$, such that if $n>N_{1}$ then $\frac{1}{1-2\left(1-\alpha_{n}\right)-b_{n}}<2$, (since $\sum_{n=1}^{\infty}\left(1-\alpha_{n}\right)^{2}<\infty$ and $\left.\sum_{n=1}^{\infty}\left(a_{i n}-1\right)<\infty\right)$. Therefore it follows from (20) that

$$
\begin{aligned}
\left\|x_{n}-p\right\|^{2} & \leq\left[1+2\left\{\left(1-\alpha_{n}\right)^{2}+b_{n}\right\}\right]\left\|x_{n-1}-p\right\|^{2} \\
& +2\left[2\left(1-\alpha_{n}\right)^{2} L \beta_{n}\left(L \beta_{n}+1\right)\right]\left\|x_{n-1}-p\right\|\left\|x_{n}-p\right\| \\
& -\left(1-\alpha_{n}\right)(1-k)\left\|x_{n}-T_{i}^{k} x_{n}\right\|^{2} \\
& -\left(1-\alpha_{n}\right)(1-k)\left\|x_{n}-T_{i}^{k} x_{n}\right\|^{2}
\end{aligned}
$$

Observe that,

$$
\begin{aligned}
\left\|x_{n}-p\right\|^{2} & =\left\langle x_{n}-p, j\left(x_{n}-p\right)\right\rangle \\
& =\alpha_{n}\left\langle x_{n-1}-p, j\left(x_{n}-p\right)\right\rangle+\left(1-\alpha_{n}\right) \\
& \cdot\left\langle T_{i}^{k} y_{n}-p, j\left(x_{n}-p\right)\right\rangle \\
& =\alpha_{n}\left\langle x_{n-1}-p, j\left(x_{n}-p\right)\right\rangle+\left(1-\alpha_{n}\right) \\
& \cdot\left\langle T_{i}^{k} y_{n}-T_{i}^{k} x_{n}, j\left(x_{n}-p\right)\right\rangle+\left(1-\alpha_{n}\right) \\
& \cdot\left\langle T_{i}^{k} x_{n}-p, j\left(x_{n}-p\right)\right\rangle \\
& \leq \alpha_{n}\left\|x_{n-1}-p\right\|\left\|x_{n}-p\right\|+L\left(1-\alpha_{n}\right) \\
& \cdot\left\|y_{n}-x_{n}\right\|\left\|x_{n}-p\right\|+\left(1-\alpha_{n}\right) L\left\|x_{n}-p\right\|^{2}
\end{aligned}
$$

Substituting (16)-(18) into (21) and simplifying this inequalities, we have

$$
\begin{aligned}
& {\left[1-\left(1-\alpha_{n}\right) L-L^{3}\left(1-\alpha_{n}\right)^{2} \beta_{n}\left(1-\beta_{n}\right)\right.} \\
& \left.-L\left(1-\alpha_{n}\right)\left(1-\beta_{n}\right)(L+1)\right]\left\|x_{n}-p\right\|^{2} \\
& \leq\left[\alpha_{n}+L\left(1-\alpha_{n}\right)^{2} \beta_{n}\left(L \beta_{n}+1\right)\right]\left\|x_{n-1}-p\right\|\left\|x_{n}-p\right\|
\end{aligned}
$$




$$
\begin{aligned}
\left\|x_{n}-p\right\| & \leq \frac{\alpha_{n}+L\left(1-\alpha_{n}\right)^{2} \beta_{n}\left(L \beta_{n}+1\right)}{1-\left(1-\alpha_{n}\right) L-L^{3}\left(1-\alpha_{n}\right)^{2} \beta_{n}\left(1-\beta_{n}\right)-L\left(1-\alpha_{n}\right)\left(1-\beta_{n}\right)(L+1)}\left\|x_{n-1}-p\right\| \\
& =\left[1+\frac{L^{3}\left(1-\alpha_{n}\right)^{2} \beta_{n}\left(1-\beta_{n}\right)+L\left(1-\alpha_{n}\right)\left(1-\beta_{n}\right)(L+1)+L\left(1-\alpha_{n}\right)^{2} \beta_{n}\left(L \beta_{n}+1\right)-\left(1-\alpha_{n}\right)}{1-\left(1-\alpha_{n}\right) L-L^{3}\left(1-\alpha_{n}\right)^{2} \beta_{n}\left(1-\beta_{n}\right)-L\left(1-\alpha_{n}\right)\left(1-\beta_{n}\right)(L+1)}\right]\left\|x_{n-1}-p\right\| \\
& \leq\left[1+\frac{L^{3}\left(1-\alpha_{n}\right)^{2} \beta_{n}\left(1-\beta_{n}\right)+L\left(1-\alpha_{n}\right)\left(1-\beta_{n}\right)(L+1)+L\left(1-\alpha_{n}\right)^{2} \beta_{n}\left(L \beta_{n}+1\right)}{1-\left(1-\alpha_{n}\right) L-L^{3}\left(1-\alpha_{n}\right)^{2} \beta_{n}\left(1-\beta_{n}\right)-L\left(1-\alpha_{n}\right)\left(1-\beta_{n}\right)(L+1)}\right]\left\|x_{n-1}-p\right\|
\end{aligned}
$$

Now, we consider the second term on the right-hand side of (23). Since $\left\{\alpha_{n}\right\}\left\{\beta_{n}\right\} \subseteq(\eta, 1)$, then

$$
\begin{aligned}
& \left(1-\alpha_{n}\right)\left[L+L^{3}\left(1-\alpha_{n}\right) \beta_{n}\left(1-\beta_{n}\right)+L\left(1-\beta_{n}\right)(L+1)\right] \\
& \leq\left(1-\alpha_{n}\right)\left[L+L^{3}+L(L+1)\right]
\end{aligned}
$$

Set $M_{2}=\left[L+L^{3}+L(L+1)\right]$. Since $\lim _{n \rightarrow \infty}\left(1-\alpha_{n}\right)=0$, then there exists a natural number $N_{2}$, such that if $n>N_{2}$ then

$$
\begin{aligned}
& 1-\left(1-\alpha_{n}\right) L-L^{3}\left(1-\alpha_{n}\right)^{2} \beta_{n}\left(1-\beta_{n}\right) \\
& -L\left(1-\alpha_{n}\right)\left(1-\beta_{n}\right)(L+1) \geq \frac{1}{2}
\end{aligned}
$$

Again it follows from the condition $\left\{\alpha_{n}\right\}\left\{\beta_{n}\right\} \subseteq(\eta, 1)$, that

$$
\begin{aligned}
& L^{3}\left(1-\alpha_{n}\right)^{2} \beta_{n}\left(1-\beta_{n}\right)+L\left(1-\alpha_{n}\right)\left(1-\beta_{n}\right)(L+1) \\
& +L\left(1-\alpha_{n}\right)^{2} \beta_{n}\left(L \beta_{n}+1\right) \\
& \leq L^{3}\left(1-\alpha_{n}\right)^{2}+L\left(1-\beta_{n}\right)(L+1)+L\left(1-\alpha_{n}\right)^{2}(L+1)
\end{aligned}
$$

Therefore it follows from (23) that

$$
\begin{aligned}
\left\|x_{n}-p\right\| & \leq\left\{1+2\left[L^{3}\left(1-\alpha_{n}\right)^{2}+L\left(1-\beta_{n}\right)(L+1)\right.\right. \\
& \left.\left.+L\left(1-\alpha_{n}\right)^{2}(L+1)\right]\right\}\left\|x_{n-1}-p\right\| \\
& =\left(1+\delta_{n}\right)\left\|x_{n-1}-p\right\|
\end{aligned}
$$

where

$\delta_{n}=2\left[L^{3}\left(1-\alpha_{n}\right)^{2}+L\left(1-\beta_{n}\right)(L+1)+L\left(1-\alpha_{n}\right)^{2}(L+1)\right]$

From conditions (ii), (iii) it is easy to see that

$\sum_{n=1}^{\infty}\left\{2\left[L^{3}\left(1-\alpha_{n}\right)^{2}+L\left(1-\beta_{n}\right)(L+1)+L\left(1-\alpha_{n}\right)^{2}(L+1)\right]\right\}$ $<+\infty$

Thus using Lemma OOA, we have $\lim _{n \rightarrow \infty}\left\|x_{n}-p\right\|$ exists, completing the proof of (i). Also it follows from (24) that $d\left(x_{n}, F\right) \leq\left[1+\delta_{n}\right] d\left(x_{n-1}, F\right)$, and it again follows from Lemma $O O A$ that $\lim _{n \rightarrow \infty}$ exists, this completes the proof of (ii).

Now, we consider the second term on the right-hand side of (21). Since $\left\{x_{n}\right\}$ is bounded, $\left\{\beta_{n}\right\} \subseteq(\eta, 1)$, then there exists a constant $M_{3}>0$ such that

$$
\begin{aligned}
& 2\left[2\left(1-\alpha_{n}\right)^{2} L \beta_{n}\left(L \beta_{n}+1\right)\right]\left\|x_{n-1}-p\right\|\left\|x_{n}-p\right\| \\
& \leq 4\left(1-\alpha_{n}\right)^{2} M_{3}
\end{aligned}
$$

Thus, it follows from (21) that

$$
\begin{aligned}
\left\|x_{n}-p\right\|^{2} & \leq\left[1+2\left\{\left(1-\alpha_{n}\right)^{2}+b_{n}\right\}\right]\left\|x_{n-1}-p\right\|^{2} \\
& +2\left(1-\alpha_{n}\right)^{2} M_{3}-\left(1-\alpha_{n}\right)(1-k)\left\|x_{n}-T_{i}^{k} x_{n}\right\|
\end{aligned}
$$

Since $\left\{x_{n}\right\}$ is bounded, then there exists a constant $M_{4}>0$ such that $\left\|x_{n}-p\right\|^{2} \leq M_{4}$. It follows from (25) that

$$
\begin{aligned}
& (1-k)\left(1-\alpha_{n}\right)\left\|x_{n}-T_{i}^{k} x_{n}\right\|^{2} \\
& \leq 2 M_{4}\left\{\left(1-\alpha_{n}\right)^{2}+b_{n}\right\}+4 M_{3}\left(1-\alpha_{n}\right)^{2} \\
& +\left\|x_{n-1}-p\right\|^{2}-\left\|x_{n}-p\right\|^{2}
\end{aligned}
$$

Hence,

$$
\begin{aligned}
& (1-k) \sum_{j=N+1}^{n}\left(1-\alpha_{j}\right)\left\|x_{j}-T_{i}^{k} x_{j}\right\|^{2} \\
& \leq 2 M_{4} \sum_{j=N+1}^{n}\left\{\left(1-\alpha_{j}\right)^{2}+b_{n}\right\}+4 M_{3} \sum_{j=N+1}^{n}\left(1-\alpha_{j}\right)^{2} \\
& +\left\|x_{N}-p\right\|^{2}<\infty
\end{aligned}
$$

Using condition (ii) and (iii), it follows from (26) that $\sum_{n=1}^{\infty}\left(1-\alpha_{n}\right)\left\|x_{n}-T_{i}^{k} x_{n}\right\|^{2}<\infty$, and using condition (i), $\liminf _{n \rightarrow \infty}\left\|x_{n}-T_{i}^{k} x_{n}\right\|=0$. Thus $\liminf _{n \rightarrow \infty}\left\|x_{n}-T_{n}^{k} x_{n}\right\|=0$.

For all $n>N$ we have $T_{n}=T_{n-N}$ so that

$$
\begin{aligned}
& \left\|x_{n}-T_{n} x_{n}\right\| \leq\left\|x_{n}-T_{n}^{k} x_{n}\right\|+\left\|T_{n}^{k} x_{n}-T_{n} x_{n}\right\| \\
& \leq\left\|x_{n}-T_{n}^{k} x_{n}\right\|+L\left\|T_{n}^{k-1} x_{n}-x_{n}\right\|
\end{aligned}
$$


Thus, $\liminf _{n \rightarrow \infty}\left\|x_{n}-T_{n} x_{n}\right\|=0$, completing the proof of (iii).

Theorem 2.1 Let $E$ be a real Banach space with normal structure $N(E)>\max \left(1, \varepsilon_{0}\right)$ and let $K$ be a nonempty closed convex subset of E. Let $\left\{T_{i}\right\}_{i=1}^{N},\left\{\alpha_{n}\right\}$, $\left\{\beta_{n}\right\}$ and $\left\{x_{n}\right\}$ be as in Lemma2.1. Then $\left\{x_{n}\right\}$ exists in $K$ and converges strongly to a common fixed point of the mappings $\left\{T_{i}\right\}_{i=1}^{N}$ if and only if

$\liminf _{n \rightarrow \infty} d\left(x_{n}, F\right)=0$ where

$$
d\left(x_{n}, F\right)=\inf _{p \in F}\left\|x_{n}-p\right\| \text {. }
$$

\section{PROOF}

The existence of fixed point follows from Theorem 1.1. If $\left\{x_{n}\right\}$ converges strongly to a common fixed point of of the mappings $\left\{T_{i}\right\}_{i=1}^{N}$, then $\liminf _{n \rightarrow \infty}\left\|x_{n}-p\right\|=0$. Since $0 \leq d\left(x_{n}, F\right) \leq\left\|x_{n}-p\right\|$, we have $\liminf _{n \rightarrow \infty}\left(x_{n}, F\right)=0$.

Conversely, suppose $\liminf _{n \rightarrow \infty}\left(x_{n}, F\right)=0$ then our Lemma implies that $\lim _{n \rightarrow \infty} d\left(x_{n}, F\right)=0$. Thus for arbitrary $\varepsilon>0$, there exists a positive integer $N_{3}$ such that $d\left(x_{n}, F\right)<\varepsilon / 4, \forall n \geq N_{3}$. Furthermore $\sum_{n=1}^{\infty} \delta_{n}<\infty$ implies that there exists a positive integer $N_{4}$ such that $\sum_{j=n}^{\infty} \delta_{j}<\frac{\varepsilon}{4 M_{4}}, \forall n \geq N_{4}$. Choose $N=\max \left\{N_{3}, N_{4}\right\}$, then $d\left(x_{n}, F\right) \leq \varepsilon / 4$ and $\sum_{j=N}^{\infty} \delta_{j} \leq \frac{\varepsilon}{4 M_{4}}$. For all $n, m \geq N$ and for all $p \in F$ we have

$$
\begin{aligned}
& \left\|x_{n}-x_{m}\right\| \leq\left\|x_{n}-p\right\|+\left\|x_{m}-p\right\| \\
& \leq\left\|x_{n}-p\right\|+M_{4} \sum_{j=N+1}^{n} \delta_{j}+\left\|x_{N}-p\right\|+M_{4} \sum_{j=N+1}^{m} \delta_{j} \\
& \leq 2\left\|x_{N}-p\right\|+2 M_{4} \sum_{j=N}^{\infty} \delta_{j}
\end{aligned}
$$

Taking infimum over all $p \in F$, we obtain

$$
\left\|x_{n}-x_{m}\right\| \leq 2 d\left(x_{N}, F\right)+2 M_{4} \sum_{j=N}^{\infty} \delta_{j} \leq \frac{2 \varepsilon}{4}+\frac{2 M \varepsilon}{4 M}=\varepsilon
$$

Thus $\left\{x_{n}\right\}$ is Cauchy. Suppose $\lim _{n \rightarrow \infty} x_{n}=u$. Then $u \in K$ since $K$ is closed. Furthermore, since $F\left(T_{i}\right)$ is closed for all $i \in I$, we have that $F$ is closed. Since $\lim _{n \rightarrow \infty} d\left(x_{n}, F\right)=0$, we must have that $u \in F$.

\section{References}

[1] M. O. Osilike, A. Udomene, D. I. Igbokwe and B. G. Akuchu, "Demiclosedness Principle and Convergence Theorems for K-Strictly Asymptotically Pseudocontractive Maps," Journal of Mathematical Analysis and Applications, Vol. 326, No. 2, 2007, pp. 1334-1345. doi:10.1016/j.jmaa.2005.12.052
[2] L. Qihou, "Convergence Theorems of the Sequence of Iterates for Asymptotially Demicontractive and Hemicontractive Mappings,” Nonlinear Analysis, Vol. 26, No. 11, 1996, 1835-1842. doi:10.1016/0362-546X(94)00351-H

[3] M. O. Osilike, S. C. Aniagboso and G. B. Akachu, "Fixed Points of Asymptotically Demicontractive Mapping in Arbitrary Banach Space,” Pan American Mathematical Journal, Vol. 12, No. 2, 2002, pp. 77-88.

[4] F. E. Browder and W. V. Petryshyn, "Construction of Fixed Points of Nonlinear Mappings in Hilbert Spaces," Journal of Mathematical Analysis and Applications, Vol. 20, No. 2, 1967, pp. 197-228. doi:10.1016/0022-247X(67)90085-6

[5] M. O. Osilike and A. Udomene, "Demiclosedness Principle and Convergence Results for Strictly Pseudocontractive Mappings of Browder-Petryshyn Type," Journal of Mathematical Analysis and Applications, Vol. 256, No. 2, 2001, pp. 431-445. doi:10.1006/jmaa.2000.7257

[6] H. Zhou, "Demiclosedness Principle with Applictions for Asymptotically Pseudocontractions in Hilbert Spaces," Nonlinear Analysis, Vol. 70, No. 9, 2009, pp. 3140-3145. doi:10.1016/j.na.2008.04.017

[7] H. K Xu and R. G. Ori, "An Implicit Iteration Process for Nonexpansive Mappings,” Numerical Functional Analysis and Optimization, Vol. 22, No. 5-6, 2001, pp. 767-733. doi:10.1081/NFA-100105317

[8] M. O. Osilike, "Implicit Iteration Process for Common Fixed Point of a Finite Family of Strictly Pseudo-contractive Maps," Journal of Mathematical Analysis and Applications, Vol. 294, No. 1, 2004, pp. 73-81. doi:10.1016/j.jmaa.2004.01.038

[9] Y. Su and S. Li, "Composite Implicit Iteration Process for Common Fixed Points of a Finite Family of Strictly Pseudocontractive Maps,” Journal of Mathematical Analysis and Applications, Vol. 320, No. 2, 2006, pp. 882-891. doi:10.1016/j.jmaa.2005.07.038

[10] Z. H. Sun, "Strong Convergence of an Implicit Iteration Process for a Finite Family of Asymptotically Quasinonexpansive Mappings," Journal of Mathematical Analysis and Applications, Vol. 286, No. 1, 2003, pp. 351-358. doi:10.1016/S0022-247X(03)00537-7

[11] M. O. Osilike and B. G. Akuchu, "Common Fixed Points of a Finite Family of Asymptotically Pseudocontractive Maps,” Journal Fixed Points and Applications, Vol. 2004, No. 2, 2004, pp. 81-88. doi:10.1155/S1687182004312027

[12] C. E. Chidume, "Functional Analysis: Fundamental Theorems with Application,” International Centre for Theoretical Physics, Trieste Italy, 1995.

[13] L. C. Ceng, H. K. Xu and J. C. Yao, "Uniformly Normal Structure and Uniformly Lipschitzian Semigroups," Nonlinear Analysis, Vol. 73, No. 12, 2010, pp. 3742-3750. doi:10.1016/j.na 2010.07.044.

[14] W. V. Petryshyn, "A Characterization of Strict Convexity of Banach Spaces and Other Uses of Duality Mappings," Journal of Functional Analysis, Vol. 6, No. 2, 1970, pp. 282-291. doi:10.1016/0022-1236(70)90061-3 